\title{
CONNOTACIONES Y PARADOJAS EN TORNO A LA PARTICIPACIÓN DE LOS ACTORES LOCALES EN INICIATIVAS DE DESARROLLO RURAL
}

\author{
Connotations and Paradoxes of Participation of Local Actors in Rural Development Initiatives
}

\author{
Ayari Genevieve Pasquier-Merino
}

Resumen: Laparticipación de los actores locales ha sido identificada como condición para mejorar la eficiencia de las iniciativas de desarrollo rural, convirtiéndose en prerrequisito de muchas de ellas, pero pocas veces se toman en cuenta sus implicaciones en lo local. En este texto se analizan algunas dinámicas sociales asociadas con la participación de los actores locales en iniciativas de desarrollo a partir de un estudio de caso realizado en una comunidad rural en la región fronteriza de Chiapas. Los argumentos incluyen que los costos del proyecto a menudo se transfieren a los participantes locales, que la condición de pobreza de los comuneros limita su participación, y que las desigualdades de poder entre los comuneros son factores determinantes para la participación.

Palabras clave: participación, proyectos de desarrollo, desarrollo rural, poder, desigualdad, pobreza.

Abstract: The involvement of local actors has been identified as a condition for improving the effectiveness and efficiency of rural development initiatives, becoming a prerequisite in many cases, but rarely taken into account are the implications of this type of intervention strategy in the local context. This article analyzes the social dynamics associated with participation of local actors in rural development intervention programs in a rural community of Chiapas located near the Guatemalan border. The arguments elaborated in the text include that costs of the projects are often transferred to local participants, that the poverty of community members severely limits their ability to participate in the projects, and that power relations of community members play a determining role in their participation.

Keywords: participation, development programs, rural development, power, inequality, poverty.

Ayari Genevieve Pasquier Merino. Doctora en Ciencias Sociales con especialidad en Sociología por El Colegio de México. Programa de Becas Posdoctorales en la Universidad Nacional Autónoma de México, becaria del Instituto de Investigaciones Antropológicas de la UNAM, México. Temas de especialización: desarrollo rural, seguridad alimentaria, gestión colectiva de recursos y migración. Correo electrónico: ayaripasquier@gmail.com.
Enviado a dictamen: 7 de abril de 2016. Aprobación: 30 de septiembre de 2016. Revisiones: 1. 


\section{Introducción}

$1 \begin{aligned} & \text { partir de la década de } 1980 \text { las agencias } \\ & \text { internacionales de desarrollo han incorporado } \\ & \text { progresivamenteplanteamientos queidentifican }\end{aligned}$ la participación de los actores locales en las intervenciones impulsadas en sus comunidades como un elemento clave para mejorar la eficiencia, eficacia y legitimidad de este tipo de estrategias (Milani, 2008). Como resultado, los beneficiarios de estos proyectos dejaron de ser considerados víctimas pasivas y fueron reconstruidos discursivamente como "socios de su propio desarrollo", lo que ha modificado las responsabilidades de las partes involucradas en estas prácticas (Dubois, 2005).

La literatura sobre desarrollo participativo ha privilegiado la documentación de los beneficios potenciales de los modelos participativos de intervención y la elaboración de metodologías y recomendaciones para mejorar su aplicación. ${ }^{1}$ En este ámbito prevalecen análisis normativos sobre quiénes deberían participar, cómo deberían organizarse, qué se busca y cómo hacerlo. La presente investigación se interesa en las construcciones discursivas respecto a la participación de los actores locales en proyectos de desarrollo rural, las prácticas operativas de iniciativas que adoptan este enfoque y sus implicaciones en las dinámicas sociales, temas que, a pesar de haber recibido poca atención, son relevantes para comprender cómo funciona este tipo de esquemas de intervención y cuáles pueden ser las precondiciones para que los actores locales se involucren en estos espacios, tomando en cuenta las particularidades contextuales.

Esta investigación se desarrolló a partir de un estudio de caso ${ }^{2}$ realizado en Nuevo Porvenir, ${ }^{3}$ una pequeña localidad ubicada en la parte baja de la zona conocida como Las Cañadas, en el municipio de Las Margaritas del estado de Chiapas, donde operaba una iniciativa de desarrollo promovida por una asociación civil llamada Kampo, que buscaba impulsar la producción agrícola comercial a través de asistencia técnica y microcrédito. ${ }^{5}$ En este contexto, en la investigación me propuse indagar: ¿cómo fueron traducidas las ideas sobre el desarrollo participativo en la operación del programa?, ¿qué incidencia tenían estas estrategias en las dinámicas sociales de la comunidad?, ¿cuáles eran los significados asociados con la participación en la esfera local?, iqué factores resultaban relevantes para comprender quiénes participaban, y de qué manera se involucraban estos actores en la iniciativa analizada?

Nuevo Porvenir estaba integrado por veinticinco familias - poco más de 150 personas-a las que Kampo dio derechos de uso sobre un predio de propiedad privada de ochenta hectáreas que la asociación compró para el desarrollo de este proyecto. El programa tenía una duración preestablecida de diez años y preveía que, al finalizar su ciclo de operación —en 2020-, la propiedad de la tierra fuera transferida a los titulares de derecho, quienes acordaron desde el inicio hacer una escritura colectiva para ahorrar costos notariales. Los integrantes de la comunidad eran en su mayoría familias originarias de Guatemala que llegaron a México como refugiados durante la década de 1980 y desde entonces habían vivido en distintas localidades de la región, buscando dónde vivir y trabajar. Aunque existían diferencias entre ellas, todas vivían en condiciones de pobreza extrema y sus modos de sustento dependían del jornaleo agrícola en los potreros y las fincas de café en la región, de las migraciones temporales de los hombres y de las transferencias gubernamentales que recibían las mujeres a través de programas sociales.

De manera esquemática puede decirse que en la región estaban presentes tres grupos sociales con historias, recursos e identidades disímiles: los rancheros mestizos, los ejidatarios -indígenas y mestizos- y los pobladores de origen guatemalteco que llegaron a México como refugiados, grupo al que pertenecían las familias de Nuevo Porvenir. Los rancheros vivían en casas con múltiples cuartos y diversas comodidades, tenían amplios predios con cultivos de autoconsumo y comerciales, algunas áreas de potrero, y muchas veces poseían una casa en la cabecera municipal y uno o más vehículos. Estas eran las personas con las que los integrantes de Nuevo Porvenir interactuaban de manera cotidiana pues eran quienes les daban empleo, les vendían productos básicos, les compraban sus cosechas y les prestaban dinero. Sus diferencias socioeconómicas 
con los ejidatarios eran menos acentuadas, aunque éstos tenían acceso a tierras y mayores servicios. Con ellos, la gente de Nuevo Porvenir realizaba pocos intercambios cotidianos.

El desarrollo de este estudio retoma la propuesta teórica de Norman Long, quien articula un esquema analítico orientado al actor para estudiar, desde un enfoque etnográfico, las interacciones entre los actores involucrados en los procesos de desarrollo. Desde esta perspectiva, las "intervenciones planeadas" son pensadas como "un proceso en movimiento, socialmente construido, negociado, experiencial y creador de significados" (Long, 2007: 65).

A modo de hipótesis se plantea que la adopción del "discurso participativo" no necesariamente conlleva la incorporación de los actores locales en la toma de decisiones y muchas veces se traduce en la implementación de estrategias que transfieren a la esfera local parte de sus costos operativos (Agudo, 2011; Molyneux, 2002), situación que resulta particularmente problemática en contextos de pobreza extrema (Meinzen-Dick y Pradhan, 2002; (Rawal, 1999). Se sugiere también que el derecho formal a participar no asegura una participación activa pues las desigualdades de recursos y poder, dentro y entre los hogares, condicionan las formas en que los actores participan -o no- en proyectos de desarrollo (Abdulwahid, 2006; Agrawal, 2010), lo que limita la inclusión de los intereses de quienes ocupan posiciones subordinadas en la planeación, regulación y distribución de recursos (Agrawal, 2010; Molyneux, 2002; Rocheleau y Edmunds, 1997); en consecuencia, la participación diferenciada puede reproducir formas de desigualdad preexistentes o generar nuevas formas de exclusión en el marco de las iniciativas de desarrollo.

El texto está organizado en cuatro secciones. En la primera se discuten distintas nociones sobre la participación de los actores locales en el ámbito del desarrollo rural, en la segunda se analizan las prácticas vinculadas con la participación en el estudio de caso, en la tercera se problematiza sobre la participación respecto a las desigualdades de poder dentro de la comunidad, y en la última se exponen algunas reflexiones sobre los costos de la participación en contextos de pobreza.

\section{Nociones sobre la participación en el ámbito del desarrollo}

El término "participación" suele ser utilizado en el ámbito del desarrollo con connotaciones positivas, ${ }^{6} \sin$ explicitarse su definición, como si fuera un concepto neutral y puramente descriptivo. Sin embargo, en las discusiones y modelos que retoman este enfoque subyacen distintas concepciones que transmiten, de manera implícita, supuestos sobre las comunidades y la manera en que se espera que los actores locales se involucren en estas iniciativas.

De manera general puede hacerse una distinción entre la participación de los actores locales como medio para llevar a cabo una intervención, es decir, como contribución en trabajo o recursos para cumplir las metas de un proyecto predefinido, y la participación como un fin en sí mismo en el marco de un proceso político que cuestiona las estructuras de poder y busca empoderar a los actores locales. Esta última perspectiva tiene sus orígenes en los trabajos de investigación realizados por Paulo Freire, Ivan Ilich, John Gaveta y Fals Borda en la década de 1970. Estos planteamientos fueron inicialmente aplicados al ámbito del desarrollo rural por algunas organizaciones no gubernamentales que plantearon promover el desarrollo de las capacidades productivas y de organización de las comunidades rurales para que ganaran autosuficiencia y mejoraran sus capacidades de demandar al Estado los servicios que requerían (Nelson y Wrights, 1995: 3).

Enlasúltimas tres décadasel "desarrolloparticipativo" ha tenido un creciente respaldo por parte de las agencias de cooperación internacional para el desarrollo, como el Banco Mundial y la Organización de las Naciones Unidas, que desde la década de 1980 promueven la idea de que los "beneficiarios" sean involucrados en la producción de sus servicios y los operadores del desarrollo se conviertan en "facilitadores" de los procesos de organización y aprendizaje locales. En este marco fueron adoptados 
los planteamientos sobre la inclusión de los actores locales en la planeación, seguimiento y evaluación de las iniciativas de desarrollo como estrategia para resolver los problemas que enfrentaban las intervenciones diseñadas desde un enfoque vertical. Los principales actores operativos de este tipo de enfoque han sido las organizaciones no gubernamentales, identificadas por la teoría de la democracia liberal y las políticas económicas neoliberales como organismos eficaces para la administración e implementación de las iniciativas de desarrollo rural (Cornwall, 2002). Este es el contexto general en el que se ha difundido el uso de las llamadas "técnicas participativas" de planeación y evaluación de proyectos, la creación de comités locales para la implementación de programas y diversos esquemas de cofinanciamiento. Para la década de 1990 estas estrategias se habían convertido en un requisito impuesto por la mayoría de los financiadores y actualmente se han integrado en la operación de los programas de política pública en diversos sectores, en algunos casos siguiendo la misma lógica de intervención vertical que este enfoque busca contrarrestar (Milani, 2008).

Desde entonces la participación ha ganado espacio en las narrativas sobre la eficacia de la gobernanza descentralizada, generando nuevos espacios oficiales de participación que han sido acompañados por transferencias de recursos y poderes en la toma de decisiones (Caragena et al. 2005; Canto, 2008; Landau, 2008); aunque éstos han tenido resultados variables (Ramos y Reyes, 2005) y no siempre responden a las necesidades e intereses de la gente (Valdivieso, 2012). Enfoques más recientes han planteado el desarrollo participativo como un proceso de reconfiguración de las estructuras regionales de gobernanza, promoviendo la creación de espacios incluyentes a través de la construcción de capacidades (McAreavey, 2009: 97); así, en vez de "invitar" a los actores locales a que participen en la planeación e implementación de intervenciones de desarrollo, se propone mejorar sus capacidades para demandar sus derechos. Sin embargo, en muchos casos este desarrollo teórico discursivo del término queda en la retórica de los documentos y no se ve reflejado en las prácticas operativas de las intervenciones en las esferas locales (Martínez y Arellano, 2007; Cardozo, 2008).

En diversos estudios se han analizado las prácticas asociadas con el "desarrollo participativo" en proyectos específicos, en los que se han hecho evidentes sus supuestos y se han mostrado las paradojas de sus dinámicas de operación y resultados. Por ejemplo, Nelson y Wrights (1995) realizaron un estudio etnográfico en el que exploraban los significados que daban a la participación los diversos actores involucrados en las intervenciones, si alguien los impugnaba y quién perdía o ganaba en estos procesos. Desde un enfoque similar, Agrawal (2010) mostró que el término "participación" hace referencia a una multiplicidad de actividades, formas de involucrarse y capacidades en la toma de decisiones. ${ }^{8}$ Por otra parte, Blackburn y Holland (1993), Cornwall (2002), Hickey y Mohan (2004), Masaki (2004) y McAreavey (2009) estudiaron la participación a partir del análisis de las interacciones sociales y el manejo de las diferencias de poder, subrayando la importancia de situar la participación en un contexto espacio-temporal específico.

Estos estudios muestran, entre otras cosas, que para entender de qué se habla cuando se hace referencia a "la participación de los actores locales en la toma de decisiones" es necesario distinguir entre recabar opiniones y transferir poderes en la toma de decisiones sobre las acciones prioritarias, cómo realizarlas y las estrategias de organización que se consideran viables.

\section{Prácticas en torno a la participación de los actores locales en iniciativas de desarrollo rural}

Los diferentes paradigmas vinculados con la participación en el ámbito del desarrollo rural suponen distintas actividades (Pacheco y Vega, 2001): la consulta sobre sus necesidades, la toma de decisiones sobre las iniciativas implementadas, la organización de actividades preestablecidas, la implementación de reglas operativas, la mano de obra en la construcción de infraestructura y el financiamiento, ${ }^{9}$ actividades que implican distintas posibilidades de inclusión y agencia de los actores locales. 
El presente estudio confirma los argumentos expuestos en otros trabajos respecto a la reformulación del discurso sobre la participación de los actores locales en términos de corresponsabilidad, acuerdos contractuales y uso de las instituciones locales y otros mecanismos sociales de organización, vigilancia y sanción para incrementar la eficiencia de los programas y mejorar el cumplimiento de sus normas (Hickey y Mohan, 2004; Mayoux, 2001). Como resultado se produce el traslado de una parte de los costos de operación hacia los "beneficiarios" (Agudo, 2011; Cooke y Kothari, 2001; Molyneux, 2002).

En los siguientes párrafos se analizan los ámbitos en los que el programa que operaba en la comunidad de estudio utilizaba estrategias participativas o de corresponsabilidad: la planeación, la legitimación de las reglas, su monitoreo, la organización y realización de actividades y elfinanciamiento de proyectos productivos.

\section{La planeación participativa}

Los ejercicios participativos de planeación han sido identificados en el ámbito del desarrollo rural como una manera de incluir a los actores locales en la definición de las prioridades y estrategias de las intervenciones de desarrollo. De acuerdo con lo anterior, el modelo de Kampo preveía la organización de "talleres comunitarios" para definir junto con los integrantes de Nuevo Porvenir su agenda anual de inversiones y actividades. En estas reuniones el personal de la asociación presentaba las iniciativas definidas previamente y no consideraba aquellas propuestas que no coincidían con su percepción de las prioridades de desarrollo de la comunidad - por ejemplo, la compra de generadores de electricidad, una de las principales demandas de las familias-.

Los ejercicios participativos de planeación pueden ser un instrumento valioso para conocer la percepción de los actores locales y tienen el potencial para abrir espacios de diálogo con personas habitualmente excluidas. Sin embargo, con base en el seguimiento del programa de Kampo en Nuevo Porvenir se plantea que su uso no siempre es sinónimo de inclusión de las perspectivas de los actores locales en los planes de intervención, porque muchas veces estos ejercicios se utilizan para validar en la esfera local iniciativas propuestas por programas definidos a priori de acuerdo con los modelos de intervención de las agencias y para legitimarlas frente a sus financiadores..$^{10}$ Esto no quiere decir que los actores locales no incidan en los proyectos desarrollados en sus comunidades pues, aunque no participen en los espacios formalmente previstos para ello, pueden incidir en éstos a través de procesos de negociación y contienda en torno a coyunturas específicas.

Por otra parte, el seguimiento de los talleres de planeación y otras reuniones organizadas por el equipo de Kampo en Nuevo Porvenir permitió documentar que una propuesta puede ser aceptada en un taller de planeación con la expectativa de recibir recursos, evitando discusiones que pueden postergarse. Pero la decisión de llevar a cabo una iniciativa, sobre todo si ésta implica inversiones de trabajo para los actores locales, debería ser entendida como un proceso influido por las interacciones y contingencias cotidianas.

Por último, con base en el análisis de los procesos de toma de decisiones ${ }^{11}$ sobre diversos proyectos en Nuevo Porvenir, parece pertinente subrayar la importancia de que los informes o estudios que utilizan ejercicios participativos de consulta o planeación hagan explícito a quiénes se refieren cuando hablan de "la comunidad", pues existen múltiples situaciones que pueden definir qué voces están siendo escuchadas, por ejemplo: iquiénes son convocados a las reuniones? -las autoridades, los titulares de derechos agrarios, toda la población, los hombres, las mujeres, los jóvenes, los integrantes de un grupo cercano a quienes organizan estos ejercicios, etcétera-; ¿quiénes pueden asistir dadas las condiciones de la reunión? - hora, lugar, duración-; zquiénes tienen el interés, la obligación o el derecho de asistir a este tipo de reuniones? y zquiénes pueden estar excluidos y por qué motivos?

De manera paralela, cabe señalar que los espacios de participación creados por agentes externos se ven afectados por las relaciones de poder en elámbito local, la posición de los actores, la organización comunitaria, sus 
capacidades de locución y otros factores. Como resultado, a pesar de los esfuerzos de los organizadores por integrar la opinión del conjunto de los asistentes, muchas veces prevalecen las voces de quienes ocupan posiciones de liderazgo. También debe tenerse en cuenta que, aun si teóricamente los operadores del programa deberían desempeñar un papel de "facilitadores" y no incidir en los ejercicios que coordinan, estas personas suelen ser vistas en el contexto local como canales de ayudas potenciales y gozan de un alto estatus - vinculado con la clase, el grado de estudios y vivir en una ciudad-, por lo que muchas veces guían la discusión mientras los "participantes" se limitan a asentir a sus propuestas. Como consecuencia, resulta fundamental que cuenten con capacidad para construir relaciones de confianza y que construyan un lenguaje común con los miembros de las comunidades donde trabajan. En este sentido se propone considerar los resultados de los ejercicios participativos de consulta como producto del encuentro entre los operadores del programa que organizan el evento y los actores locales (Villarreal, 1994).

\section{La legitimación de las reglas}

Las relaciones entre el equipo de Kampo y los integrantes de Nuevo Porvenir estaban enmarcadas por un conjunto de reglas operativas establecidas por la asociación que se encontraban enumeradas en contratos individuales entre Kampo y cada uno de los titulares de derecho de la comunidad. Estas reglas fueron legitimadas a través de una serie de acuerdos inicialmente pactados con la asamblea comunitaria y, para mejorar su cumplimiento, el personal de Kampo se apoyaba en las figuras de autoridad reconocidas localmente. Sin embargo, ninguno de estos mecanismos se traducía en su cabal cumplimiento. El respaldo de las autoridades y la asamblea a las reglas de Kampo no era incondicional y en ciertas coyunturas resultaba frágil, sobre todo cuando se hizo evidente que el personal del programa no podía imponer el cumplimiento de las reglas del programa sin el respaldo de las autoridades comunitarias. Cuando surgía algún desacuerdo era común que se realizaran reuniones en las que las partes exponían sus argumentos, pero se enfrentaban grandes problemas para construir acuerdos duraderos, situación que resultaría en una creciente tensión. Esta dinámica muestra la relevancia de los argumentos expuestos por Long (2007) respecto al carácter activo de los actores locales y la naturaleza dinámica de las intervenciones de desarrollo.

\section{El monitoreo de las reglas y la organización de actividades}

Para el monitoreo de las reglas y la organización de las actividades previstas por el programa, Kampo retomó la figura tradicional del sistema de cargos y pidió a la asamblea comunitaria que se designaran "comisiones" ad hoc para dar seguimiento a tareas específicas - construcción de infraestructura, seguimiento de trabajos agrícolas, etcétera-; de esta manera, trasladó a la esfera local la responsabilidad sobre algunas tareas vinculadas con la operación de sus iniciativas. El funcionamiento de estas comisiones enfrentaba diversos problemas. El equipo de Kampo se quejaba de la falta de compromiso de los integrantes de las comisiones, y en general tenía dificultades para coordinarse con ellos. Por su parte, varios miembros de la comunidad señalaban que el personal de Kampo no respetaba los acuerdos iniciales y remplazaba sus funciones.

\section{El financiamiento de los proyectos productivos}

El financiamiento de los proyectos que llevaba a cabo Kampo en Nuevo Porvenir estaba basado en dos esquemas: la contribución de los actores locales en la construcción de infraestructura y el microcrédito. En ambos casos se observó el uso de las instituciones locales y el traslado de una parte de los costos asociados con la operación de una intervención a la esfera local.

En el primer caso, Kampo utilizaba un esquema común en muchos programas dirigidos a la construcción de infraestructura, donde se preveía que los actores externos financiaran los materiales y la asesoría técnica a cambio de que los actores locales contribuyeran con su mano de obra. Esta estrategia retoma la figura tradicional 
del "trabajo comunitario", una contribución ocasional y no remunerada utilizada en muchas comunidades rurales para hacer frente a obras de beneficio común. En Nuevo Porvenir, los miembros de la asamblea preferían evitar este tipo de estrategias, pues participar en estas iniciativas les hacía dejar otras labores pendientes. Además, con frecuencia surgían desacuerdos sobre cómo y cuándo realizar las obras, lo que evidenciaba que el equipo de Kampo y los integrantes de la comunidad tenían lógicas distintas. Los operadores de Kampo respondían a los planes de trabajo definidos por sus superiores, a los presupuestos aprobados para cada mes y a los reportes de actividades que debían entregar. Pero cuando llegaban a la comunidad debían adaptar su agenda a los tiempos y modos de los integrantes de Nuevo Porvenir, quienes modificaban los acuerdos formalizados en el plan de trabajo anual según las necesidades y oportunidades que surgían día con día.

La falta de coordinación y la escasa disposición de tiempo por parte de los titulares de derecho de la comunidad solía llevar a retrasos en la realización de los trabajos y en general limitaba la construcción de las obras de uso común financiadas por Kampo. Esta situación contradecía la visión del personal de la asociación, que resaltaba la importancia de sus propuestas y se decía sorprendido por la negativa de los integrantes de la comunidad a aprovechar que alguien financiara la construcción de infraestructura y les otorgara capacitación técnica.

El análisis del microcrédito como estrategia de cofinanciamiento resulta más complejo. La promoción de estas estrategias en contextos rurales se basa en la idea de que el microcrédito permite que los pequeños productores mantengan sus niveles de consumo a lo largo del proceso de producción, hagan inversiones para impulsar la producción agropecuaria de pequeña escala y diversifiquen sus modos de sustento, lo que reduciría su vulnerabilidad y mejoraría sus condiciones de vida. En diversos estudios se ha documentado la centralidad de las prácticas financieras vinculadas con el crédito en la economía familiar y comunitaria (González de la Rocha, 2006; Long, 2007; Villarreal 2004; Guérin, 2011). Sin embargo, en un creciente número de estudios se argumenta que el endeudamiento incrementa la vulnerabilidad de las familias pobres, ${ }^{12}$ lo que se ha documentado con base en casos en los cuales el aumento de la oferta de microcrédito rural dirigido a pequeños productores ha creado nuevas "trampas de pobreza" y conflictos dentro de las comunidades (Guérin et al., 2009; Morvant-Roux, 2009; Bateman, 2012; Taylor, 2012; Marr, 2012).

Los integrantes de Nuevo Porvenir conocían los riesgos que implicaba el endeudamiento y, a pesar de que el equipo de Kampo les aseguraba que si trabajaban bien sus cafetales no tendrían problemas, describían la deuda como una gran preocupación y un peso.

Uno de los argumentos más usados para explicar el fracaso de los proyectos de microcrédito es que los recursos destinados a la inversión productiva se integran a la lógica de la economía familiar, donde las inversiones compiten con las necesidades cotidianas del hogar. Pero este no es el único problema que enfrenta el microfinanciamiento en contextos rurales (Collins y Morduch, 2011). Un microcrédito no basta para que pequeños productores en condiciones de pobreza puedan integrarse a un mercado competitivo. Múltiples condiciones restringen la diversificación de actividades económicas y su rentabilidad, entre las que destacan la marginación geográfica, la falta de infraestructura y la discriminación social (Marr, 2012; Harper, 2012; Shaw, 2004). Además, las actividades agropecuarias enfrentan grandes riesgos que aumentan la vulnerabilidad de los deudores (Duvendack et al., 2011) y la presión de pago puede afectar la economía de los hogares (Marr, 2012; Korth et al., 2012; Bateman, 2012; Harper, 2012) e incrementar la desigualdad (Taylor, 2012).

Con base en lo anterior, algunos autores han sugerido la necesidad de flexibilizar los esquemas utilizados por las instituciones de microfinanza y difundir servicios financieros paralelos al crédito, en particular el ahorro y los seguros (Marr, 2012; Montgomery, 1996). El caso de Nuevo Porvenir confirma que el impulso de actividades agropecuarias a través del microcrédito enfrenta retos que rebasan la falta de servicios financieros, también muestra que las instituciones de microfinanza pueden adaptarse a las condiciones de la producción 
agropecuaria, pero no pueden cambiar aquellos factores contextuales que limitan el desarrollo y reproducen la desigualdad en ausencia de un política articulada ${ }^{13}$ que favorezca la distribución de oportunidades y riqueza (Shaw, 2004; Zeller et al., 1997).

Por último, vale la pena señalar que los riesgos vinculados con los proyectos de microcrédito afectan sobre todo a las familias más pobres, quienes pueden verse obligadas a usar estos recursos para el consumo y luego vender sus activos para pagar las deudas, además de que, por tener un menor poder de negociación, suelen ser objeto con mayor frecuencia de las sanciones previstas por el incumplimiento de los pagos (Dichtery Harper, 2007; Mosley y Hulme, 1998; Guérin et al., 2009; Guérin, 2011; Mosley, 2001; Montgomery, 1996; Shaw, 2004). En el caso de estudio esta situación llevaría a la exclusión de las familias más vulnerables del programa.

\section{Participación, desigualdad y relaciones de poder}

Los espacios creados por las iniciativas de "desarrollo participativo" están marcados por una serie de supuestos sobre las comunidades y la participación que definen en gran medida quién y cómo participa, sin considerar las diferencias de poder en las esferas locales, diferencias que han sido ignoradas por gran parte de la literatura en torno al desarrollo participativo (Cooke y Kothari, 2001), al suponer de manera implícita una serie de condiciones que coinciden con la concepción de Jürgen Habermas sobre la "esfera pública" como un espacio de deliberación horizontal, donde la toma de decisiones está basada en la construcción de consensos. Este supuesto pasa por alto que las prácticas relacionadas con el desarrollo participativo están inmersas en los juegos políticos del contexto local (Cooke y Kothari, 2001; Blauert, Anta y Graf, 2006), donde suelen ser reproducidas las inequidades de estatus y posición social (Finn, 1994; Kohn, 2000). Los espacios que enmarcan la participación de los actores locales en las iniciativas de desarrollo nunca son neutrales; en ellos participan actores que tienen diferentes posiciones e intereses, y desiguales capacidades de maniobra. Cuando se habla de "la comunidad", sus decisiones y sus preferencias, es fundamental tener en cuenta las divergencias internas y las desigualdades de poder.

En este apartado se analiza la operación cotidiana de las desigualdades de poder en la toma de decisiones colectivas sobre la gestión de los recursos llevados a la comunidad de estudio por la iniciativa de desarrollo que ahí operaba - principalmente tierras donde vivir y trabajar e insumos agrícolas-. Con este fin, se dio seguimiento a distintos procesos de construcción de consensos y toma de decisiones en el marco de la asamblea comunitaria.

En la esfera local la asamblea era considerada como máxima autoridad ${ }^{14}$ e identificada como un espacio de deliberación horizontal. Formalmente todos sus integrantes podían expresarse libremente ${ }^{15}$ y las decisiones eran formalizadas a través de la votación de "acuerdos comunitarios". Sin embargo, a lo largo del estudio se documentó la prevalencia de las perspectivas sostenidas por las personas con mayor liderazgo. Con frecuencia había quien argumentaba abiertamente objeciones a las propuestas de los líderes y en ocasiones esto abría cierto marco de negociación. No obstante, muchos miembros de la comunidad compartían la sensación de que esto fuera inútil, e incluso contraproducente, pues generalmente daba pie a sanciones implícitas que se traducían en una presión continua sobre los actores que habían mostrado su inconformidad. ${ }^{16}$ En los hechos la asamblea comunitaria era un espacio de negociación entre actores con capacidades desiguales de incidir en la toma de decisiones, pero la imagen de la asamblea como un espacio de construcción consensuada de acuerdos borraba el carácter negociado, y a menudo conflictual, de la toma de decisiones, limitando las posibilidades de disentimiento de las voces críticas.

En el análisis se identificaron diversos tipos de participación en función de los derechos y responsabilidades de los integrantes de la comunidad y su capacidad de agencia. Para organizarlos se retoma la propuesta de Agrawal (2010), quien distingue la participación en la toma de decisiones como: nominal - pertenecer a un grupo-, pasiva - acudir 
a reuniones sólo para escuchar las decisiones que se toman o enterarse de las decisiones por otros mediosconsultativa - solicitud de opinión no vinculante sobre cuestiones específicas-, activa específica - realizar tareas predefinidas - activa - expresar opiniones, sean o no requeridas, o tomar otro tipo de iniciativas-e interactiva - tener voz e influencia en las decisiones de un grupo, ocupar posiciones- (Agrawal, 2010: 172).

Con base en este esquema se identifican tres patrones a través de los cuales operaba la desigualdad en la asamblea de Nuevo Porvenir: tener derecho formal de participar, pero estar excluido de las situaciones donde se tomaban las decisiones - que era el caso de las mujeres-; tener derecho formal de participar y estar presente en las discusiones, pero no sentirse legitimado para participar — que era el caso de los jóvenes-; tener derecho formal de participar en la toma de decisiones, estar presente en las reuniones y participar de manera activa en las discusiones, pero no contar con las capacidades para construir consensos en torno a sus posturas.

Los liderazgos en Nuevo Porvenir estaban asociados con una cierta disponibilidad de recursos económicos y sociales, pero en gran medida se construían cotidianamente y dependían de las capacidades personales de ciertos actores para presentarse como una guía eficaz, conveniente y necesaria para todos. Estas personas tenían el respaldo incondicional de sus grupos parentales y de quienes esperaban obtener beneficios a cambio de su apoyo - por ejemplo, evitar sanciones-. Estos liderazgos fueron reforzados por la intervención de Kampo, pues inicialmente el personal de la asociación buscó su apoyo para facilitar la operación del programa y la construcción de acuerdos ofreciéndoles a cambio múltiples beneficios. La contribución de intervenciones externas en la reproducción de las relaciones de exclusión existentes y el incremento de la desigualdad en las comunidades ha sido ampliamente documentada por la literatura (Nuijten, 2003; Ribot y Larson 2005; Cornwall 2002; Navarro, Cejudo y Maroto, 2014). Argumentaciones similares se harían en su tiempo respecto a los esquemas de "gobierno indirecto" utilizados por las administraciones coloniales.
Respecto al papel del género en el análisis sobre la desigualdad en la toma de decisiones, el presente estudio documenta que, aun si las mujeres tenían derechos formales para participar en la toma de decisiones colectivas, las decisiones que tenían que ver con la operación de Kampo eran tomadas en juntas extraordinarias a las que ellas no asistían. Además, la jerarquía en las relaciones de género las situaba en una posición subordinada frente a los hombres, quienes eran identificados como los legítimos tomadores de decisiones en nombre de todos los miembros de sus hogares. Contrario a lo que sucede en otras comunidades (Bonfil, Barrera y Aguirre, 2008), en Nuevo Porvenirlas mujeres no parecían estar interesadas en participar en los espacios colectivos, que consideraban como esferas masculinas, y aceptaban a los hombres como representantes de los intereses del hogar.

Las iniciativas de desarrollo rural que incluyen un "enfoque participativo" suelen homologar a sus beneficiarios, obviando las complejidades y tensiones subyacentes al funcionamiento de los espacios colectivos (Milani, 2008). El análisis desarrollado en Nuevo Porvenir sugiere que la creación de espacios para la participación de los actores locales en las iniciativas de desarrollo no asegura mayor equidad en la distribución de poderes y recursos, pues las jerarquías de la comunidad están presentes en estos espacios y pueden utilizarse para reforzar las relaciones de poder y exclusión existentes. Como señala Andrea Cornwall:

Power relations pervade any spaces for participation. Spaces made available by the powerful may be discursively bounded to permit only limited citizen influence, colonising interaction and stifling dissent. Spaces fostered as a way of amplifying marginalised voices may end up being filled by gatekeepers, who speak for but not with those they represent; attempts at the widest possible involvement can go awry when noone can be bothered to take part. [...] And participatory processes may serve simply to reproduce echoes of dominant knowledges rather than to amplify the alternative, "bottom-up" perspectives that are claimed for them (Cornwall, 2002: 8-9). ${ }^{17}$ 
Para complementar el análisis sobre la desigualdaden Nuevo Porvenir, se indagó también sobre su incidencia en las prácticas cotidianas de uso y apropiación de los recursos puestos a disposición de los miembros de la comunidad por el programa de Kampo, en particular de la tierra y los insumos agrícolas, que eran las principales áreas de acción del programa. Entre los factores más relevantes para explicar las diferencias en el uso de estos activos destacan la composición y el ciclo vital de las familias, que definía la disponibilidad de mano de obra, su capacidad productiva y sus posibilidades de convertir los recursos provenientes de la iniciativa en beneficios a mediano y largo plazo. Teniendo en cuenta esto, las familias más jóvenes enfrentaban condiciones particularmente difíciles. Otro factor relevante era el tipo de empleo que desempeñaban los hombres fuera de la comunidad. Quienes lograban emplearse como peones en la construcción ganaban entre 200 y 250 pesos diarios, mientras los jornaleros agrícolas ganaban 50 pesos. El trabajo que se desempeñaba dependía de las experiencias previas y de las redes sociales de cada familia. Contar con un poco más de ingresos permitía a estas personas contratar ayudantes en las labores agrícolas cuando debían enfrentar tareas pesadas y durante sus ausencias, sin que sus necesidades de subsistencia los obligaran a dejar de lado estas actividades, además de poder invertir en insumos agrícolas para mejorar sus cosechas.

En el presente estudio se sugiere que, cuando una iniciativa de desarrollo ignora las asimetrías sociales existentes en una comunidad, corre el riesgo de reproducirlas, o incluso acentuarlas, excluyendo a los actores más vulnerables, quienes según los discursos de la mayor parte de las agencias deberían ser los beneficiarios privilegiados de estas iniciativas (Cleaver, 2005; Gardner y Lewis, 2003; Mosse, 2008; Guijt y Shah, 1998).

\section{Los costos de la participación en contextos de pobreza}

Como se dijo al inicio del texto, en las últimas décadas se ha enfatizado la importancia de que los actores locales participen en la planeación, implementación y evaluación de los proyectos de desarrollo rural y emprendan procesos organizativos para facilitar la satisfacción de necesidades que requieren activos difíciles de obtener de manera individual en contextos de pobreza (Di Gregorio et al., 2008; Woolcock y Narayan 2000). Sin embargo, pocas veces se tiene en cuenta cómo inciden las condiciones de pobreza en las formas en que se involucran los actores locales en las iniciativas propuestas por entes externos como estrategias para mejorar sus vidas.

El estudio de caso desarrollado en Nuevo Porvenir documenta que la participación en las actividades previstas por el modelo de Kamporesultaba costosa para los actores locales, sobre todo en tiempo - asistencia a reuniones, pláticas informativas, talleres, visitas y recorridos-, un recurso escaso en un contexto en el que la mayoría de los esfuerzos de las familias estaban destinados a cubrir los gastos cotidianos de subsistencia.

El equipo de la asociación consideraba la asistencia a estos eventos como contraparte por los beneficios que daban a la comunidad; para los miembros de la comunidad, asistir a estas actividades podía traducirse en la pérdida de un día de jornal o en el retraso en sus cultivos, y con frecuencia buscaban deslindarse o simplemente no asistían. ${ }^{18}$

Ocupar un cargo en alguna de las comisiones previstas por el programa era la forma de participación más dispendiosa, por el tiempo invertido pero también porque los cargos estaban asociados con una serie de expectativas por parte del resto del grupo y del personal de la asociación. Quienes ocupaban o habían ocupado estas posiciones coincidían en que su trabajo implicaba una fuerte presión social y mucho trabajo que no era reconocido. Además, las posiciones requeridas por el programa se sumaban a las posiciones asociadas con la estructura local de gobernanza, lo que saturaba el sistema de cargos local.

Los costos asociados con la participación en el caso de estudio tenían una distribución desigual. Las mujeres y los jóvenes - no titulares de derecho-, excluidos de los espacios de organización comunitaria, estaban exentos de las labores vinculadas a las 
iniciativas de Kampo. Entre los titulares de derecho existían desigualdades importantes en la distribución de cargas de trabajo, que dependían de la función que desempeñaba cada uno de ellos, pero sobre todo de su compromiso en el cumplimiento de las tareas.

En la presente investigación se sugiere que, en condiciones de pobreza, las actividades vinculadas con iniciativas de desarrollo pueden entrar en conflicto con las labores cotidianas de las cuales depende el sustento de las familias, lo que puede dejar fuera a las personas más necesitadas o restringir la viabilidad de las iniciativas promovidas. De manera similar, pareciera existir un umbral de pobreza que limita las posibilidades de beneficiarse de proyectos de microcrédito (Cleaver, 2005) y de involucrarse en iniciativas colectivas que tienen el potencial de reducir la vulnerabilidad de los hogares (De Haan, 2001; Uphoff y Wijayaratna, 2000).

\section{Conclusiones}

La vigencia y difusión del paradigma de la participación social en el desarrollo responde a múltiples experiencias que han mostrado que las iniciativas de desarrollo impulsadas por actores externos que se mantienen a mediano y largo plazo son aquellas en las que los actores locales han estado involucrados y se han apropiado de cada una de las etapas de los proyectos implementados (Diego, 2008).

La presente investigación forma parte de un creciente número de estudios en los que se analizan iniciativas gubernamentales y no gubernamentales que incorporan la participación como parte de su diseño operativo, documentando que en muchos casos se transfieren responsabilidades a los actores locales en una búsqueda de mejorar la eficiencia y reducir los costos asociados con estas iniciativas, pero no se les hace partícipes de la toma de decisiones vinculadas con la definición de las prioridades, soluciones y estrategias de las intervenciones promovidas en sus comunidades.

Almismotiempo, elanálisisdeestetipodeexperiencias muestra el carácter activo de los actores locales y pone en evidencia que las intervenciones planeadas no son un proceso lineal definido únicamente por objetivos y reglas preestablecidas, sino el resultado de procesos de negociación ${ }^{19}$ en los que se modifican los esquemas inicialmente propuestos y se visibilizan las divergencias de significado e intereses entre las agencias y los actores locales (Long, 2007). Estas negociaciones dialécticas se configuran como un espacio donde pueden analizarse los mecanismos a través de los cuales las intervenciones externas pueden reforzar las diferencias de poder y perpetúan un sistema clientelar (Cooke y Kothari, 2001). En este sentido, el estudio de caso desarrollado en Nuevo Porvenir muestra la importancia de que las iniciativas que incluyen esquemas participativos tengan en consideración no sólo los beneficios, sino también los costos que implica su intervención para los actores locales, y presten atención a posibles patrones de exclusión vinculados con sus diseños operativos.

Por otra parte, la presente investigación argumenta la pertinencia de incluir, en los resultados de los ejercicios participativos llevados a cabo en este ámbito, información que permita conocer quién ha participado, cómo han participado los actores involucrados, qué relaciones de poder y dominación pudieron haber incidido en la definición de los participantes y su modo de participar, quién o quiénes pudieron estar excluidos y quién se ha beneficiado del proceso, dejando de lado los supuestos sobre "la comunidad" como un espacio horizontal y homogéneo.

De manera más general, los argumentos presentados a lo largo del texto plantean algunas otras interrogantes sobre la participación de los actores locales en iniciativas de desarrollo, por ejemplo: iqué se busca al promover este tipo de esquemas?, ¿qué formas de participación prevén los programas impulsados?, ¿qué precondiciones existen para que los actores locales participen en éstos? y, más en general, icuáles son las condiciones para que los espacios creados para la participación de los actores locales en iniciativas de desarrollo rural puedan convertirse en oportunidades reales para que ganen derechos y adquieran herramientas para mejorar sus vidas? Se trata de preguntas de las que resulta necesario seguir indagando frente a la creciente presencia de los esquemas participativos en los programas y políticas públicas. 


\section{Notas}

${ }^{1}$ Este tipo de investigaciones han sido promovidas por grandes instituciones de desarrollo, como el Banco Mundial y las Naciones Unidas, interesadas en promover enfoques participativos de desarrollo.

${ }^{2}$ Para esta investigación se dio seguimiento al proyecto de Kampo en Nuevo Porvenir durante el periodo 20102013; el primer año se realizó una visita breve y se mantuvo contacto a distancia con el personal de Kampo, en el periodo 2011-2013 se hicieron diversas estancias de dos o tres semanas en la comunidad. En la recopilación de datos se privilegiaron técnicas etnográficas, de manera complementaria se construyó una base de datos con información de los hogares.

${ }^{3}$ Los nombres de lugares, organizaciones y personas fueron sustituidos por seudónimos para preservar la privacidad de las personas, siguiendo el acuerdo al que se llegó en la comunidad al inicio de la investigación.

${ }^{4}$ Esta asociación funcionaba con recursos provenientes de una asociación norteamericana que desde hace más de treinta años ha financiado proyectos similares en diversos países centroamericanos. En México, Kampo estaba presente en cinco comunidades de la región fronteriza del estado de Chiapas, operando con distintos esquemas.

${ }^{5}$ La operación de Kampo en Nuevo Porvenir estaba centrada principalmente en el desarrollo de cultivos comerciales de café y plátano, pero también incluía el desarrollo de cultivos de autoabasto y pequeñas actividades familiares como la cría de aves de corral y el cultivo de hortalizas.

${ }^{6}$ En general la participación se considera como fundamento de la democracia e indicador de una sociedad equitativa. Esta connotación tiene sus orígenes en los ideales de una sociedad integrada, en la que los individuos están vinculados a una colectividad por un sentido de pertenencia y buscan el bien común.

7 Distinción utilizada por Nelson y Wright (1995), Blackburn y Holland (1993) y Cornwall (2002), entre otros.

${ }^{8}$ Respecto a la planeación del trabajo, la decisión de las reglas de uso de los recursos, la especificación e implementación de penalidades por el rompimiento de las reglas, la organización de las estrategias de monitoreo, el trabajo de provisión, la distribución de los beneficios, las estrategias de mercado, la resolución de conflictos a varias escalas y la gestión de los fondos.

${ }^{9}$ Clasificaciones de los tipos de participación en los programas de desarrollo pueden encontrarse en: Canto (2008), Michener (1998) y Oakley (1991).

${ }^{10}$ Estos argumentos coinciden con otros trabajos en los que se analizan prácticas similares en diversos contextos, por ejemplo: Cooke y Kothari (2001), Gardner y Lewis (2003), Hickey y Mohan (2004) y Mayoux (2001).

${ }^{11}$ Este análisis se basa en el seguimiento de las reuniones de planeación, organización y realización de diversas iniciativas.

${ }^{12}$ Esta vulnerabilidad está asociada con las condiciones de su contexto, las fluctuaciones de la economía global, las necesidades vinculadas con el ciclo de vida, situaciones de enfermedad o muerte de algún miembro de la familia y malas cosechas, entre otros factores.

${ }^{13}$ Que incluyera: una reforma agraria, la protección de la agricultura frente a las importaciones subsidiadas, la inversión pública en infraestructura, obras y derechos de irrigación, precios mínimos garantizados, protección de los recursos comunes, respaldo de los derechos de los jornaleros frente a los grandes propietarios y contratistas, y formas subsidiarizadas de crédito para los pequeños propietarios.

${ }^{14} \mathrm{El}$ marco normativo de la comunidad identificaba como miembros de la asamblea a todos los hombres titulares de un derecho sobre la tierra, considerados localmente como legítimos tomadores de decisiones. Kampo intentó impulsar la participación de las mujeres en las asambleas, pero estas asistían sólo a las reuniones más importantes y por lo general no participaban de manera activa.

${ }^{15}$ A lo largo del estudio se documentó el uso de distintas estrategias discursivas para buscar el respaldo colectivo, por ejemplo: explicar los beneficios o problemas que ocasionaría la aprobación de una iniciativa, haciendo referencia a una lógica de cálculo costo-beneficio; hacer mención a las normas y acuerdos de la comunidad, retomando el derecho local como argumento; exponer las razones por las que un planteamiento 
debía ser considerado como más justo, siguiendo una lógica moral; explicar las condiciones personales que llevaban a sostener cierta postura, buscando la empatía de la audiencia; o deslegitimar las propuestas de otros miembros de la asamblea asociándolos con características negativas - como la violación de normas o la falta de trabajo- y diversos tipos de reclamos y presiones.

${ }^{16}$ Este ejemplo coincide con el estudio de Cleaver (2005).

17 "Las relaciones de poder impregnan cualquier espacio para la participación. Los espacios creados por los poderosos pueden estar delimitados discursivamente para permitir que sólo algunos ciudadanos incidan, controlando las interacciones y reprimiendo la disidencia. Los espacios promovidos como maneras de amplificar las voces de los marginados pueden ser ocupados por "controladores de acceso", que hablan a nombre de, pero no junto con quienes representan; los intentos por un involucramiento lo más amplio posible pueden fracasarcuando nadie puede ser molestado para participar. [...] y los procesos participativos pueden servir simplemente para reproducir las perspectivas de los conocimientos dominantes en vez de promover las perspectivas alternativas provenientes de las bases como se supone que hacen" (traducción propia).

${ }^{18}$ El reglamento de la comunidad preveía el cobro de multas por faltar a las actividades convocadas por Kampo. Según me comentó el agente municipal, todos los miembros de la comunidad habían acumulado adeudos por este concepto, pero era imposible cobrarles pues no tenían dinero y la asamblea justificaba estas faltas.

${ }^{19}$ En este marco es pertinente resaltar el papel mediador de los "operadores del desarrollo", quienes adaptan los proyectos definidos por las agencias en las que trabajan a las realidades cambiantes del campo. Según sugiere Long (2007: 149-182), estos actores ocupan una posición de "administradores de interfaz" por ser parte de una realidad técnica e institucional que enmarca sus actividades y responsabilidades y, al mismo tiempo, están comprometidos con las dinámicas y problemas sobre los que busca incidir el programa en que trabajan.

\section{Referencias bibliográficas}

Abdulwahid, Saratu (2006). "Gender Differences in Mobilization for Collective Action: Case Studies of Villages in Northern Nigeria". Capri Working Paper 58. Washington, D.C.: CGIAR.

Agrawal, Bina (2010). Gender and Green Governance. The Political Economy of Women's Presence Within and Beyond Community Forestry. Oxford: Oxford University Press.

Agudo Sanchíz, Alejandro (2011). "Mejoras privadas, beneficios colectivos: la producción y subversión de regímenes globales de política social en Chiapas". En Alejandro Agudo Sanchíz y Marco Estrada Saavedra (eds.), (Trans)formaciones del Estado en los márgenes de Latinoamérica. Imaginarios alternativos, aparatosinacabados yespacios transnacionales. México: El Colegio de México /Universidad Iberoamericana.

Bateman, Milford (2012). "The Role of Microfinance in contemporary Rural Development Finance Policy and Practice: Imposing Neoliberalism as "Best Practice". En Journal of Agrarian Change 12(4): 587-600.

Blackburn, James, y Jeremy Holland (eds.) (1993). Who Changes? Institutionalizing participation in development. Londres: Intermediate Technology Publications.

Blauert, Jutta, Martha Rosas, Salvador Anta y Sergio Graf (2006). “iEspacios para la deliberación o la toma de decisiones? Lecciones para la participación y las políticas en Consejos ambientales en México". En Ernesto Isunza Vera y Alberto J. Olvera (coords.), Democratización, rendición de cuentas y sociedad civil: participación ciudadana y control social. México: H. Cámara de Diputados, LIX Legislatura/CIESAS/ Universidad Veracruzana /Miguel Ángel Porrúa, pp. 596-693.

Bonfil Sánchez, Paloma, Dalia Barrera Bassols e Irma Aguirre Pérez (2008). "Los espacios conquistados. Participacion politica y liderazgo de las mujeres indígenas de México". México: Programa de las Naciones Unidas para el Desarrollo.

Canto, Manuel (2008). "Gobernanza y participación ciudadana en las políticas públicas frente al reto del desarrollo". En Politica y Cultura, 30: 9-37. 
Cardozo Brum, Myriam (2008). "Gestión y evaluación participativas en políticas sociales”. En Política y Cultura, 30: 137-63.

Cartagena Ticona, Ruth Pamela, Manuel Roberto Parra Vázquez, Araceli Burguete Cal y Mayor y Antonio López Meza (2005). "Participación social y toma de decisiones en los consejos municipales de desarrollo rural sustentable de Los Altos de Chiapas". En Gestion y Politica Publica, 14(2): 341-402.

Cleaver, Frances (2005). "The Inequality of Social Capital and the Reproduction of Chronic Poverty". En World Development, 33(6): 893-906.

Collins, Darly y Jonathan Morduch (2011). Las finanzas de los pobres. Cómo viven los pobres del mundo con dos dólares al día. México: Random House Mondadori.

Cooke, Bill, y Uma Kothari (eds.) (2001). Participation: The New Tyranny? Nueva York: Zed Books.

Cornwall, Andrea (2002). "Making spaces, changing places: situating participation in development". IDS Working Paper 170.

De Haan, Nicoline (2001). "Of Goats and Groups: A Study on Social Capital in Development Projects". En Agriculture and Human Values, 18(1): 71-84.

Dichter, Thomas y Malcolm Harper (eds.) (2007). What's Wrong with Microfinance? Warwickshire: Practica Action Publishing.

Diego Quintana, Roberto Serafín (2008). "Participación y empoderamiento a partir de experiencias de desarrollo rural en México: icual es la cuestión”. En Política y Cultura, 30: 193-208.

Dubois, Alfonso (2005). "Equidad, bienestar y participación. Bases para construir un desarrollo alternativo". En Cuadernos de Trabajo Hegoa, 26: 1-64.

Duvendack, Maren, Richard Palmer-Jones, James G. Copestake y Lee Hooper (2011). "What is the Evidence of the Impact of Microfinance on the Well-Being of Poor People?" Londres: PPI-Centre/Social Science Research Unit/Institute of Education-University of London.

Finn, Janet L. (1994). "The Promise of Participatory Research". En Journal of Progressive Human Services, 5(2): 25-42.

Gardner, Katy y David Lewis (2003). Antropología, desarrollo y el desafío posmoderno. Cuernavaca: UNAM-
CRIM/El Colegio de México/Universidad Autónoma de Chapingo.

German, Laura, Hailemichael Taye, Sarah Charamila, Tesema Tolera y Joseph Tanui (2008). "The Many Meanings of Collective Action - Lessons on Enhancing Gender Inclusion and Equity in Watershed Management". CAPRI Working Paper, 17. Washington D.C.: CGIAR/IFPRI.

González de la Rocha, Mercedes (2006). Procesos domésticos y vulnerabilidad. Perspectivas antropológicas de los hogares con Oportunidades. México: Centro de Investigaciones y Estudios Superiores en Antropología Social.

Gregorio, Monica Di, Political Science, y Konrad Hagedorn (2008). "Property Rights, Collective Action, and Poverty: The Role of Institutions for Poverty Reduction". En CAPRI Working Paper, 81. Washington D.C.: CGIAR/IFPRI.

Guérin, Isabelle (2011). "L'expérience vécue de la microfinance. Appropriations, tensions et recompositions des rapports sociaux". En Cultures e Conflits, 83: 39-59.

Guerin, Isabelle, Marc Roesch, Santosh Kumar, Venkatasubramanian y Mariam Sangare (2009). "Microfinance and the Dynamics of Financial Vulnerability. Lessons from Rural South India". Marsella: Rural Microfinance and Employment Project. Guijt, Irene y Meera Kaul Shah (eds.) (1998). The Myth of Community: Gender Issues in Participatory Development. Londres: Intermediate Technology Publications.

Harper, Malcolm (2012). "Microfinance Interest Rates and client Returns". En Journal of Agrarian Change, 12(4): 564-574.

Hickey, Samuel y Giles Mohan (2004). Participation: form Tyranny to Transformation? Exploring New Approaches to Participation in Development. Nueva York: Zed Books.

Kohn, Margaret (2000). "Language, Power, and Persuasion: Toward a Critique of Deliberative Democracy". En Constellations, 7(3): 408-429.

Korth, M., R. Stewart, C. Van Rooyen y T. de Wet (2012). "Microfinance: Development Intervention or Just Another Bank?" En Journal of Agrarian Change, 12(4): 575-586. 
Landau, Matías (2008). "La participación en las políticas públicas y los límites de la metáfora espacial”. En Política y Cultura, 30: 67-89.

Long, Norman (2007). Sociologíadel desarrollo:unaperspectiva centrada en el actor. San Luis Potosí/Ciudad de México: El Colegio de San Luis/Centro de Investigaciónes y Estudios Superiores en Antropología Social.

Marr, Ana (2012). "Effectiveness of rural Microfinance: What We Know and What We Need to Know". En Journal of Agrarian Change, 12(4): 555-563.

Martínez Ibarra, Jorge Arturo y Rosa Elena Arellano Montoya (2007). "Participación social y desarrollo rural sustentable en la microcuenca Lagunillas, Jalisco, México". En Cuadernos de Desarrollo Rural, 58(59): 49-64.

Masaki, Katsuhiko (2004). "The 'transformative' Unfolding of 'Tyrannical' Participation: the Corvèe Tradition and Ongoing Local Politics in Western Nepal". En Samuel Hickey y Giles Mohan (eds.), Participation: from Tyranny to Transformation? Exploring New Approaches to Participation in Development. Londres: Zed Books.

Mayoux, Linda (2001). "Tackling the Down Side: Social Capital, Women's Empowerment and Micro-Finance in Cameroon". En Development and Change, 32(3): 435-464.

McAreavey, Ruth (2009). Rural Development Theory and Practice. Nueva York: Routledge.

Michener, Victoria J. (1998). "The participatory Approach: Contradiction and Co-option in Burkina Faso". En World Development, 26(12): 2105-2118.

Milani, Carlos R.S. (2008). "Discursos y mitos de la participación social en la cooperación internacional para el desarrollo: una mirada a partir de Brasil". En Revista Española de Desarrollo y Cooperación, 22: 161-181.

Molyneux, Maxine (2002). "Gender and the Silences of Social Capital: Lessons from Latin America". En Development and Change, 33(2): 167-188.

Montgomery, Richard (1996). "Disciplining or Protecting the Poor? Avoiding the Social Costs of peer Pressure in micro-Credit Schemes". En Journal of International Development, 8(2): 289-305.

Morvant-Roux, Solène (2009). “Accès au microcrédit et continuité des dynamiques d'endettement au
Mexique: Combiner anthropologie économique et économétrie”. Francia: Université Lumière Lyon 2.

Mosley, Paul (2001). "Attacking Poverty and the 'PostWashington consensus"'. En Journal of International Development, 13(3): 307-313.

Mosley, Paul y David Hulme (1998). "Microenterprise Finance: Is There a Conflict between Growth and Poverty Alleviation?" En World Development, 26(5): 783-790.

Mosse, David (2008). "Authority, Gender and Knowledge: Theoretical Reflections on the Practice of Participatory Rural Appraisal". En Development and Change, 25(3): 497-526.

Navarro, Francisco, Eugenio Cejudoy Juan Carlos Maroto (2014). "Reflexiones en torno a la participación en el desarrollo rural. ireparto social o reforzamiento del poder?" En Eure, 40(121): 75-91.

Nelson, Nici y Susan Wrights (eds.) (1995). Power and Participatory Development: Theory and Practice. Londres: Intermediate Technology.

Nuijten, Monique (2003). Power, Community and the State. The Political Anthropology of Organisation in Mexico. Londres: Pluto Press.

Oakley, Peter (ed.) (1991). Projects with People: The Practice of Participation in Rural Development. Ginebra: International Labour Office.

Pacheco Vega, Raúl y Obdulia Vega López (2001). "Dos modalidades de participación ciudadana en política ambiental". En Economía, Sociedady Territorio, III(9): 25-61.

Ramos, José María y Marcela Reyes (2005). "Gobiernos locales y participación ciudadana: Hacia un enfoque de gestión estratégica asociada". En Espiral, XII(34): 39-66.

Rawal, Vikas (1999). "Participation of the Rural Poor in Dairy Cooperatives. Case Studies from Gujarat". Roma: Rural Household Income Atrategies for Poverty Alleviation and Interactions with Local Institutional Environment.

Ribot, Jesse (2004). Democratic Decentralization Of Natural Resources: Encountering And Countering Resistance. Washington D.C.: World Resources institute.

Ribot, Jesse C. y Anne M. Larson (eds.) (2005). Democratic Decentralization Of Natural Resources: Encountering And Countering Resistance. Londres: Routledge. 
Rocheleau, Dianne y David Edmunds (1997). "Women, Men and Trees: Gender, Power and Property in Forest and Agrarian Landscapes". En World Development, 25(8): $119-140$.

Shaw, Judith (2004). "Microenterprise Occupation and Poverty Reduction in Microfinance Programs: Evidence from Sri Lanka”. En World Development, 32(7): 1247-1264.

Taylor, Marcus (2012). "The Antinomies of financial Inclusion': Debt, Distress and the Workings of Indian Microfinance". En Journal of Agrarian Change, 12(4): 601-610.

Uphoff, Norman y C.M. Wijayaratna (2000). "Demonstrated Benefits from Social Capital: The Productivity of Farmer Organizations in Gal Oya, Sri Lanka". En World Development, 28(11): 1875-1890.

Valdivieso, Patricio (2012). "Políticas públicas de participación ciudadana". En Debates, 1(6): 149-171.
Villarreal, Magdalena (1994). Wielding and Yielding: Power, Subordination and Gender Identity in the Context of a Mexican Development Project. Holanda: Wageningen University.

Villarreal, Magdalena (2004). Antropología de la deuda. Crédito, ahorro fiado y prestado en las finanzas cotidianas. México: Centro de Investigaciones y Estudios Superiores en Antropología Social.

Woolcock, Michael y Deepa Narayan (2000). "Social Capital: Implications for Development Theory, Research, and Policy". En The World Bank Research Observer, 15(2): 225-249.

Zeller, Manfred, Gertrud Schrieder, Joachim Von Braun y Franz Heidhues (1997). "Finance for Food Security for the Poor. Implications for research and policy". 4. Food Policy Reviews. Washington D.C. 